POS $\quad$ PROCEEDINGS

\title{
Neutrinos from the Fermi Bubbles with ANTARES
}

\author{
Steffen Hallmann* \\ Universität Erlangen-Nürnberg, Erwin-Rommel-Str. 1, 91058 Erlangen, Germany \\ Erlangen Centre for Astroparticle Physics (ECAP) \\ E-mail: steffen.hallmann@fau.de \\ Thomas Eberl \\ Universität Erlangen-Nürnberg, Erwin-Rommel-Str. 1, 91058 Erlangen, Germany \\ Erlangen Centre for Astroparticle Physics (ECAP) \\ E-mail: thomas.eberlefau.de
}

\section{on behalf of the ANTARES Collaboration}

The Fermi Bubbles are giant lobe-shaped structures of $\gamma$-ray emission extending above and below the Galactic Center. A variety of both hadronic and leptonic models has been proposed to explain the emission, yet its origin remains unclear. Only in hadronic scenarios, the $\gamma$-ray flux is accompanied by an associated neutrino flux that might be detected with large volume neutrino detectors. ANTARES is the largest neutrino telescope in the Northern Hemisphere and has been operated in its final configuration since 2008. Its location in the Mediterranean sea provides an excellent view towards the Fermi Bubbles' region. In a previous analysis ANTARES has observed a nonsignificant excess of neutrinos in the Fermi Bubble region using charged current interactions of $v_{\mu}$. For these events, the outgoing muon produces an elongated track-like signature that can be reconstructed with sub-degree angular resolution. More recently, improved shower reconstruction algorithms achieve a precision of $\sim 3^{\circ}$ also for signatures produced in $v_{e}, v_{\tau}$ and neutral current interactions. This contribution presents the search for shower-like events from the Fermi Bubbles in ANTARES data. In combination with the previous analysis, upper limits on the all-flavour neutrino emission from the region are derived.

35th International Cosmic Ray Conference - ICRC2017

10-20 July, 2017

Bexco, Busan, Korea

* Speaker. 


\section{The Fermi Bubbles - giant lobes of $\gamma$-rays and neutrinos?}

The Fermi Bubbles are two giant areas of bright high-energy $\gamma$-ray emission extending $50^{\circ}$ above and below the Galactic Center. Although the structure has been first observed in FermiLAT data in 2010 [1, 2], its origin is still not unambiguously identified. Most of the proposed emission scenarios suggest $\gamma$-ray generation by either leptons $[3,4,5]$ or hadrons $[6,7,8]$. In hadronic scenarios, high energy protons, diffused from the environment of the Galactic Center, produce charged mesons and subsequently $\gamma$-rays upon interaction with the ambient gas. In this case the decays of (mainly) charged pions give rise to an accompanying neutrino flux that can be searched for with large-volume neutrino detectors like the ANTARES telescope. In case the origin is leptonic, the $\gamma$-ray flux from synchrotron emission of high energy $e^{ \pm}$lacks a neutrino counterpart. Ultimately, (non-)observation of a neutrino flux from the Fermi Bubbles can therefore constrain the hadronic component of the $\gamma$-ray flux.

ANTARES [9] is a neutrino telescope located at the bottom of the Mediterranean Sea in a depth of $2.5 \mathrm{~km}$. It consists of 885 optical modules (OMs) distributed over twelve detection lines that register Cherenkov radiation induced by charged secondary particles emerging from neutrino interactions in the sea water. In the detector, neutrinos can have two main event topologies. In charged-current (CC) interactions of high energy $v_{\mu}$, the produced muon travels several hundred meters in sea water while emitting Cherenkov light. The detected signature in the optical sensors appears therefore track-like. The second topology has a more contained emission - showerlike events - and is produced by CC interactions of $v_{e}$ and neutral-currents of all flavours. The tau-lepton produced in CC interactions of $v_{\tau}$ decays rapidly and mostly gives rise to shower-like signatures. Only in its decay to a muon (17\%), the event may appear track-like.

Track signatures have already been analysed in a previous analysis [10] in the data recorded with ANTARES between May 2008 and December 2015. Here, we present the search for showerlike events in the same period and derive combined all-flavour upper limits on the Fermi Bubble neutrino flux.

\section{Shape of the bubbles and analysis strategy}

The measured $\gamma$-ray flux is to first order uniformly bright throughout the extension of the bubbles. When discovered, a hard spectrum close to $\approx E^{-2}$ with no clear cutoff up to several hundred $\mathrm{GeV}$ was observed by Fermi-LAT [2]. With higher statistics and after more detailed analysis, either a sub-TeV cutoff or a significant softening at high energies is meanwhile preferred [11]. The shape of the Fermi Bubbles is shown in Fig. 1 (left). While a tentative flux-intense 'cocoon' shape in the central region has been suggested earlier on [11], it was only recently that the Fermi Bubbles have been analysed at latitudes below $10^{\circ}$ [12]. Here, a relatively hard $\sim E^{-2.1}$ with no cutoff up to several $100 \mathrm{GeV}$ is observed. Also, the $\gamma$-ray flux in the low-latitude region is three times higher compared to the rest of the bubbles. This might be a hint of a change in the composition of the Fermi Bubbles towards low latitudes.

However, as the possible emission region of neutrinos is not known a priori, the same simplified hourglass shape as in the track analysis is used as signal region. The background in this on-zone 

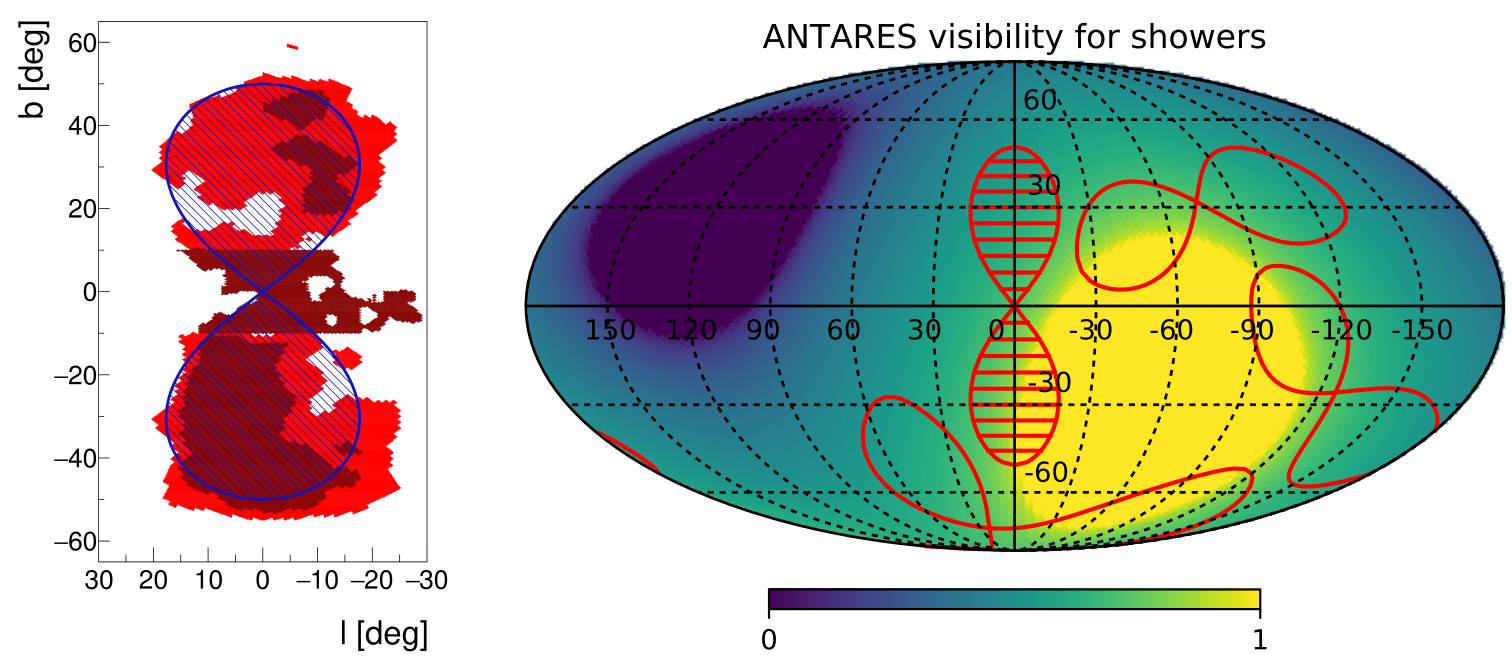

Figure 1: Left: Shapes of the Fermi Bubbles' templates observed at high and low latitudes by Fermi-LAT $[11,12]$. The hourglass shape used in the analysis is indicated in blue. Right: On- and off-zones in Galactic coordinates. The color code shows the visibility for events in the shower analysis.

is estimated from three off-zones shifted in time by $1 / 4,1 / 2$ and $3 / 4$ sidereal days to ensure similar visibility for each zone (see Fig. 1 (right)).

\section{Assumed spectrum and flux}

For the emission we assume a uniform $\gamma$-ray flux of

$$
E^{2.18} \frac{\mathrm{d} \Phi_{\gamma}}{\mathrm{d} E}=(0.5-1.0) \times 10^{-6} \mathrm{GeV}^{1.18} \mathrm{~cm}^{-2} \mathrm{~s}^{-1} \mathrm{sr}^{-1}
$$

throughout the signal shape, corresponding to an $\sim E^{-2.25}$ proton flux [13]. Previously, ANTARES tested also a harder $E^{-2} \gamma$-ray spectrum. However such a spectrum is meanwhile significantly constrained by the HAWC telescope unless for very low cutoff energies in the GeV domain [14]. As shown in Fig. 2, high cutoff energies are meanwhile disfavoured even for the softer spectrum.

For the softer spectral index, the corresponding neutrino (anti-neutrino) flux per flavour in a purely hadronic scenario is a factor $0.188(0.175)$ smaller than the $\gamma$-ray emission given neutrino oscillation has equalised the flavour composition $v_{e}: v_{\mu}: v_{\tau}=1: 1: 1$ at the detector [15].

Within our galaxy sources are not expected to efficiently accelerate protons beyond $1-10 \mathrm{PeV}$ in energy [6]. This induces also a cutoff in the observed $\gamma$-ray and neutrino spectra. As an approximation $20 \%$ of the proton energy is on average converted into charged pions. Equipartition of this energy over the four daughters in pion decay yields $E_{\text {cutoff }, v}=0.05 \times E_{\text {cutoff }, p}$ for neutrinos, i.e. optimistic cutoff energies ranging from 50 to $500 \mathrm{TeV}$.

Hence, the probed neutrino fluxes per flavour are:

$$
E^{2.18} \frac{\mathrm{d} \Phi_{\text {model }}}{\mathrm{d} E}=(1.8-3.6) \times 10^{-7} \mathrm{GeV}^{1.18} \mathrm{~cm}^{-2} \mathrm{~s}^{-1} \mathrm{sr}^{-1} \times \exp \left(-\frac{E}{E_{\text {cutoff }, v}}\right),
$$

with $E_{\text {cutoff }, v}=[\infty, 500 \mathrm{TeV}, 100 \mathrm{TeV}, 50 \mathrm{TeV}]$. 


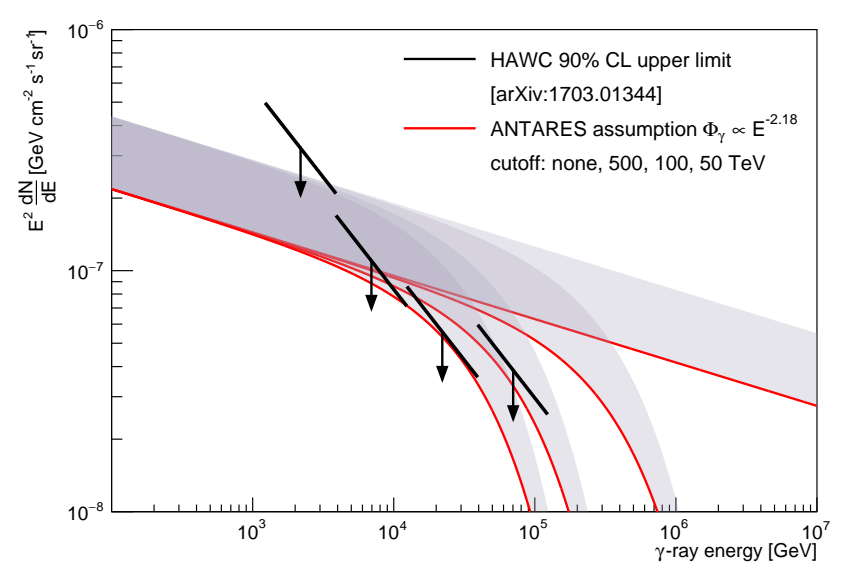

Figure 2: Assumed $\gamma$-ray flux bands (c.f. eq. 3.1) and HAWC upper limits [14] on the northern bubble. Conservative lower bounds (red lines) were used for the optimisation of the shower event selection. The same cutoff values as assumed for neutrinos are shown for illustration.

\section{Shower analysis: Event reconstruction and selection}

For the shower analysis, all recorded runs with stable data-taking conditions are selected. In the period from March 2008 to December 2015 a total livetime of 2097 days is used.

Shower-like events in ANTARES are reconstructed with a recently developed algorithm [16] in two subsequent steps. First, the shower vertex is determined by minimising a robust M-estimator, $M_{\text {est }}$, using the charge and time of signal-like photon hits on the OMs. In a second step, the obtained vertex and the amplitudes registered by the OMs are used to perform a maximum likelihood fit of the shower direction and to estimate the neutrino energy, $E_{\text {shower }}$. With this algorithm a directional resolution of $\lesssim 3^{\circ}$ is achieved for shower events.

Most of the reconstructed events in ANTARES are of atmospheric origin. Despite the overburden of sea water, muons produced in cosmic ray air showers can reach the detector from above. Therefore only event directions from below or close to the horizon, $\cos \left(\right.$ zenith $\left._{\text {shower }}\right)<0.1$, are accepted. Since many mis-reconstructed atmospheric muons have a fitted vertex far outside the detector, events reconstructed close to the instrumented volume are selected, in particular the radial and vertical distance from the detector center are restricted to $R_{\text {shower }}<300 \mathrm{~m}$ and $\left|z_{\text {shower }}\right|<250 \mathrm{~m}$, respectively. To reduce the contribution from atmospheric muons and badly reconstructed events in the analysis further, a series of additional selection cuts are applied. These are a cut on the $M_{\text {est }}$ parameter, a cut on the shower likelihood ratio, $L_{\text {shower, }}$ which uses information of the shower hits used in the fit, a cut on the angular error estimate $\beta_{\text {shower }}$ of the shower reconstruction and cuts on two additional quality parameters from other algorithms. The latter two are the quality parameter of a grid-scan based track reconstruction algorithm, $R_{\text {GridFit }}$, and the output variable of a random decision forest (RDF) trained on the fit result of a second shower reconstruction algorithm [17]. In addition, only events that did not pass the final event selection of the track analysis $[18,10]$ are accepted to provide two disjoint event samples.

Atmospheric neutrinos may only be discriminated from a Fermi Bubble signal thanks to their soft $\sim E^{-3.7}$ energy spectrum. As a consequence a lower cut on the energy estimate $E_{\text {shower }}$ is applied. The full set of cuts is listed in Tab. 1.

Whilst some of the cuts serve only for a loose de-selection of badly reconstructed events, others are optimised by using the dedicated Monte Carlo simulation that is generated for each data-taking 
Table 1: List of selection cuts for shower events. Further details on the parameters are given in the text. Cuts labeled with $(*)$ have been optimised to yield the optimal sensitivity for a neutrino cutoff at $50 \mathrm{TeV}$. The others have been applied for pre-selection.

\begin{tabular}{|c|c|c|c|c|}
\hline parameter & cut value & & & \\
\hline \multirow{6}{*}{$\begin{array}{l}R_{\text {shower }} \\
\left|z_{\text {shower }}\right| \\
M_{\text {est }} \\
\text { GridFit_R } \\
\text { does not pa }\end{array}$} & \multirow{6}{*}{$\begin{array}{l}<300 \mathrm{~m} \\
<250 \mathrm{~m} \\
<1000 \\
>0.7 \\
\text { s track selection }\end{array}$} & \multirow{2}{*}{$\begin{array}{l}\cdots \\
\cos \left(\text { zenith }_{\text {shower }}\right)\end{array}$} & & \multirow{2}{*}{$(*)$} \\
\hline & & & $\begin{array}{l}<0.1 \\
<30^{\circ}\end{array}$ & \\
\hline & & $\beta_{\text {shower }}$ & $<30^{\circ}$ & (*) \\
\hline & & $R D F$ & $>0.4$ & $(*)$ \\
\hline & & $L_{\text {shower }}$ & $>20$ & $(*)$ \\
\hline & & $E_{\text {shower }}$ & $>2290 \mathrm{GeV}$ & $(*)$ \\
\hline
\end{tabular}

run in ANTARES to account for the variable environmental conditions and the state of the detector. In the available Monte Carlo simulation, the $v_{\tau}$ channel was not simulated on a run-by-run basis. The contribution of $v_{\tau}$ was included by scaling the $v_{\mu} \mathrm{CC}$ and $v_{e} \mathrm{CC}$ and $\mathrm{NC}$ ratios adequately to take into account the $v_{\tau} \rightarrow \mu, v_{\tau} \rightarrow e, v_{\tau} \rightarrow$ hadron contributions. [19]

The cuts on $\cos$ (zenith shower $), \beta_{\text {shower }}, L_{\text {shower }}, R D F$ and $E_{\text {shower }}$ have been optimised to yield the most stringent average $90 \%$ upper limit on the Fermi Bubble flux,

$$
\bar{\Phi}_{90 \%}=\Phi_{\text {model }} \times \frac{\bar{s}_{90 \%}(b)}{s} .
$$

Here, $s$ is the number of simulated and selected signal neutrinos generated and reconstructed within the signal region, and $b$ is the average over simulated background events reconstructed within the off-zone regions. The average signal upper limit,

$$
\bar{s}_{90 \%}(b)=\sum_{k=0}^{\infty} \mu_{90 \%}(k, b) \times \text { Poisson }(k \mid b),
$$

is summed over Feldman-Cousins signal upper limits [20] with a $90 \%$ confidence level, $\mu_{90 \%}$, for any possible event count in the on-zone, $k$, weighted with the respective Poisson probability. In the optimisation only the cut on the shower energy variable depends strongly on the assumed signal cutoff. $E_{\text {shower }}>2.29 \mathrm{TeV}$ is optimal for the $50 \mathrm{TeV}$ cutoff scenario and chosen as selection cut for the analysis. All other selection cuts applied are listed in Tab. 1. The number of background events per off-zone expected from Monte Carlo simulation is 8.9. Table 2 summarises the flux sensitivities and corresponding signal expectations for different cutoffs. The sensitivity in the shower channel alone is approximately an order of magnitude above the assumed flux. The sensitivity of ANTARES worsens significantly for even lower cutoff energies due to the dominant atmospheric neutrino flux at low energies.

\section{Observed shower events}

Good agreement of data and Monte Carlo simulation in the off-zones has been verified both with the loose pre-selection cuts and with the final selection excluding the cut on $E_{\text {shower }}$ for all selection variables used. In addition, to ensure that the same number of events can be expected 
Table 2: Flux sensitivities in units of the model flux per flavour and expected number of signal events for different cutoff values of the neutrino flux.

\begin{tabular}{r|rrrr}
\hline Cut-off energy & $\infty$ & $500 \mathrm{TeV}$ & $100 \mathrm{TeV}$ & $50 \mathrm{TeV}$ \\
\hline sensitivity / $\Phi_{v_{\alpha}+\bar{v}_{\alpha}}$ & 7.2 & 8.7 & 12 & 15 \\
signal (Monte Carlo) & 0.90 & 0.75 & 0.55 & 0.44 \\
\hline
\end{tabular}

from all analysis zones, it has been checked using a variety of randomly chosen selection cut values that the simulated event counts agree on the percent level between on- and the average over the off-zones and that the off-zone difference of data events is within the expectation from statistical fluctuations.

Applying the event selection described above, a total of 40 events are observed in the three off-zones, resulting in an expected background of 13.3 events for the signal region. Here, 16 events are detected. This corresponds to a small and non-significant excess of $0.6 \sigma$ in the shower channel alone using the method from Ref. [21]. The $E_{\text {shower }}$ distribution of the events in the on- and offzones is presented in Fig. 3. Observed event counts in the zones slightly exceed the Monte Carlo expectation indicated as green band. To account for flux uncertainties, an additional $30 \%$ error on the neutrino flux is included in the error bands. In the selected event sample the statistics of simulated atmospheric muons is very low, which might explain the small excess of events seen in data with respect to the simulations. For the measurement however, the background is determined from off-zones without use of Monte Carlo simulations. Here, the event distribution in the on-zone is in good agreement with the off-zones.

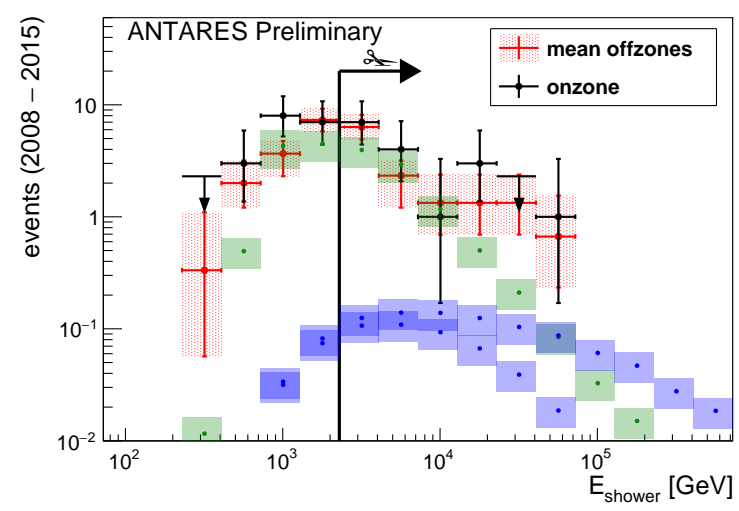

Figure 3: Energy distibution of data (including $1 \sigma$ Poisson intervals) in the shower analysis with the event selection applied. The sum of the simulated Monte Carlo expectation from atmospherics and the possible Fermi Bubble flux with $50 \mathrm{TeV}$ cutoff and no cutoff are shown as green and blue bands, respectively. The cut on the energy estimate $E_{\text {shower }}>2.29 \mathrm{TeV}$ is indicated.

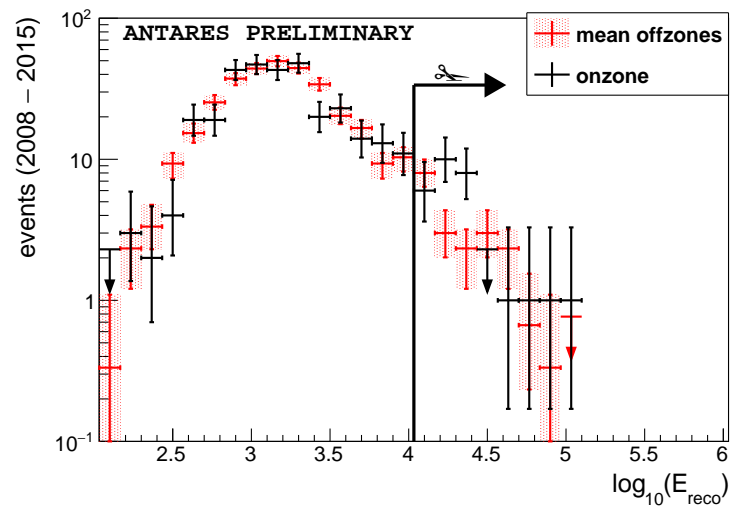

Figure 4: Energy distrubution for data from the onand off-zones in the track analysis (including $1 \sigma$ Poisson intervals) after event selection. The lower cut on the energy estimate $E_{\text {reco }}>10^{4.03} \mathrm{GeV}$ is indicated. [10] 


\section{Summary of the track reconstruction and results}

The previous analysis of the track channel in Ref.[10] analysed the same period from May 2008 to end 2015. Also in the track channel, a non-significant $(1.5 \sigma)$ excess of 28 events was observed compared to an average number of 19.7 events seen inside the off-zones. Figure 4 shows the event distribution in the track channel. The visibility for the Fermi Bubbles in the track analysis is slightly reduced compared to showers, due to a zenith angle cut exactly at the horizon which is needed to limit the contribution from mis-reconstructed atmospheric muons.

\section{All-flavour upper limits}

The small over-fluctuations in the shower and the track analysis are non-significant. Therefore the on- and offzone counts of the track and shower analyses $\left(N_{\mathrm{on}, \mathrm{t} / \mathrm{s}}, \bar{N}_{\mathrm{off}, \mathrm{t} / \mathrm{s}}\right)$ are used to derive combined $90 \%$ upper limits on the Fermi Bubble neutrino flux:

$$
\Phi_{90 \%}=\Phi_{\text {model }} \times \frac{\mu_{90 \%}\left(N_{\text {on }, \mathrm{t}}+N_{\mathrm{on}, \mathrm{s}} \mid \bar{N}_{\mathrm{off}, \mathrm{t}}+\bar{N}_{\mathrm{off}, \mathrm{s}}\right)}{s_{\mathrm{t}}+s_{\mathrm{s}}} .
$$

Resulting upper limits are put in the region where the central $90 \%$ of the possible signal from the track and shower channel $\left(s_{\mathrm{t} / \mathrm{s}}\right)$ overlap in Fig. 5. Note that a detailed study of systematic uncertainties is ongoing and not yet included in the presented upper bounds. The bounds do not reach the sensitivity of ANTARES (cf. Tab. 2) due to the small excesses seen in both channels. The combined upper limits are a factor 0.72 (no cutoff) to 0.61 (50 TeV cutoff) more stringent than the upper limits obtained from the track analysis alone.

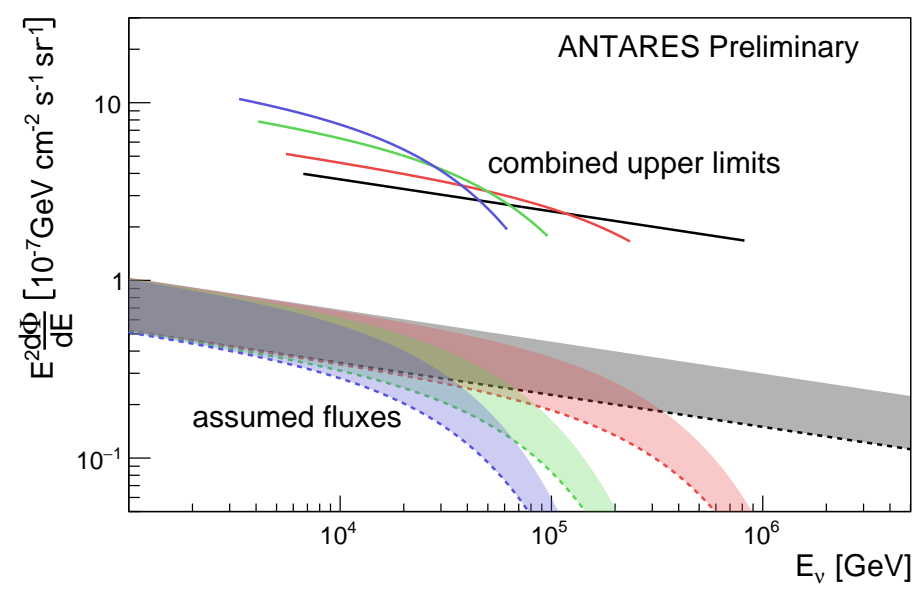

Figure 5: Combined 90\% upper limits on the neutrino flux from the Fermi Bubbles for $50 \mathrm{TeV}, 100 \mathrm{TeV}, 500 \mathrm{TeV}$ and no cutoff. Upper limits are restricted to the regions where the central $90 \%$ of signal from the track and shower channel overlap.

\section{Conclusion}

With the recent upper limits on the high energy photon flux from the Fermi Bubbles, optimistic assumptions for the neutrino flux to which ANTARES is most sensitive are disfavoured. The derived combined upper limits are an order of magnitude above the tested flux with a softer spectral index. Also, no evidence for a neutrino excess from the Fermi Bubbles region is observed in IceCube data $[22,23]$. In future, the KM3NeT detector under construction in the Mediterranean 
Sea will improve the event statistics and the sensitivity in the region of the bubbles [24]. Especially an extension to very high energies of the recently identified hard flux component at low latitudes [12] - which unfortunately is outside the field of view of the HAWC observatory - can be probed with neutrinos using the KM3NeT detector.

\section{References}

[1] G. Dobler, D. P. Finkbeiner, I. Cholis, T. Slatyer, and N. Weiner ApJ 717 (July, 2010) 825-842, [arXiv:0910.4583].

[2] M. Su, T. Slatyer, and D. Finkbeiner ApJ 724 (2010) 1044, [arXiv: 1005.5480 ].

[3] P. Mertsch and S. Sarkar Phys. Rev. Lett. 107 (2011) 091101, [arXiv: 1104.3585$].$

[4] G. Dobler, I. Cholis, and N. Weiner ApJ 741 (2011) 25, [arXiv: 1102.5095 ].

[5] H.-Y. K. Yang and M. Ruszkowski ArXive-prints (June, 2017) [arXiv: 1706.0502 ].

[6] R. Crocker and F. Aharonian Phys. Rev. Lett. 106 (2011) 101102, [arXiv:1008. 2658].

[7] S. Thoudam ApJ, Letters 778 (2013) L20, [arXiv: 1304 . 6972].

[8] B. C. Lacki Monthly Notices of the RAS 444 (2014) L39, [arXiv: 1304 . 6137].

[9] M. Ageron and et al. NIM A 656 (Nov., 2011) 11-38, [arXiv: 1104 . 1607].

[10] T. Eberl and S. Hallmann for the ANTARES Coll. Proceedings of the Neutrino 2016 (2016).

[11] M. Ackermann et al. ApJ 793 (2014) 64, [arXiv: 1407 . 7905].

[12] The Fermi-LAT Collaboration ArXiv e-prints (Apr., 2017) [arXiv:1704.0391].

[13] S. R. Kelner, F. A. Aharonian, and V. V. Bugayov Phys. Rev. D 74 (2006), no. 3034018.

[14] A. U. Abeysekara et al. ApJ 842 (2017) 2, 85.

[15] F. L. Villante and F. Vissani Phys. Rev. D 78 (2008) 103007, [arXiv: 0807.4151$].$

[16] T. Michael, ANTARES Coll. Proceedings of the 34th ICRC PoS (ICRC2015) 1078 (2015).

[17] ANTARES Collaboration ArXiv e-prints (Mar., 2017) [arXiv:1703.0243].

[18] S. Adrian-Martinez et al. Eur. Phys. J. C74 (2014) 2701, [arXiv: 1308 . 5260].

[19] ANTARES Collaboration ArXiv e-prints (2017) [arXiv:1706.0185].

[20] G. J. Feldman and R. D. Cousins Phys. Rev. D 57 (1998) 3873, [physics / 9711021 ].

[21] T.-P. Li and Y.-Q. Ma ApJ 272 (1983) 317.

[22] K. Fang, M. Su, T. Linden, and K. Murase ArXiv e-prints (2017) [arXiv:1704.0386].

[23] N. Sherf, U. Keshet, and I. Gurwich ArXiv e-prints (2017) [arXiv:1705.0666].

[24] KM3NeT Collaboration, S. Adrian-Martinez et al. ApJ 42 (2013) 7. 